Scripta METALLURGICA et MATERIALIA
Vo1. 30, pp. 95-100, 1994

Printed in the U.S.A.
Pergamon Press Ltd.

All rights reserved

\title{
DEFORMATION BEHAVIOR OF A 70/30 ALPHA BRASS IN PLANE STRESS/ PLANE STRAIN
}

\author{
P.A. Sundaram, D. Rodriguez* and S. Santiago* \\ Department of Mechanical Engineering \\ *Department of Electrical Engineering \\ University of Puerto Rico \\ Mayaguez, Puerto Rico, US 00680
}

(Received September 27, 1993)

\section{Introduction}

Generally a simple power law $\sigma=\mathrm{K} \varepsilon^{\mathrm{n}}$ (where $\mathrm{K}$ and $\mathrm{n}$ are empirical constants called the strength coefficient and the work hardening coefficient respectively) is used to describe plastic stress-strain dependence of deformable metals $(1,2)$. This equation was employed by Low (3) to describe the uniaxial tensile behavior of an annealed 70/30 brass. The slope of the corresponding double logarithmic true stress-true strain plot gives a single value of $n$. However, this fit is not always adequate. Geil and Carwile (4) reported a deviation in the linearity of the double logarithmic plot for steel. Two sets of $\mathrm{K}$ and $\mathrm{n}$ values were used to describe the stress-strain relationship in a spheroidized hypo-eutectoid steel by Wilson and Konnan (5). Various reasons, such as grain size effects, cementite particle distribution, cell formation, stacking fault energy, etc. were proposed to rationalize the double- $n$ behavior (6-9). Ollilainen reported a triple- $n$ behavior for a decarburized steel (10).

A study by Valkonen (11) of the mechanical behavior of a Clausing specimen was carried out for an AISI 1090 spheroidized steel. A good dimensional description of the Clausing specimen is given elsewhere (12). Fig. 1 shows the geometry and dimensions of the specimen. The deformation field in the gage section of the specimen on applying a uniaxial tensile load is described as plane stress/plane strain. The extent of these states depends on the width to thickness ratio of the specimen (13). The results of Valkonen indicate that the above steel shows a multiple-n type behavior. Three slope discontinuities were presented in the double logarithmic true stress vs. true strain plot. Concurrently, five slope discontinuities were presented in a "unified" plot of the double logarithmic engineering stress vs. true strain plot. Various reasons, relating to both material and constitutive properties, were hypothesized as to the appearance of these discontinuities. Saturation back stress at second phase carbide particles soon after yielding, self-development of a lateral constraint, the nucleation and growth of incipient shear bands etc. were given as plausible reasons.

Although the observation of multiple-n behavior has been previously reported in many cases (4-11), it has not found wide acceptance, probably because of a lack of concrete experimental evidence. This work was undertaken in order to investigate further the presence of these slope discontinuities and determine possible reasons for their appearance. 70/30 $\alpha$ brass was chosen as the test material. $\alpha$ brass also shows a propensity for the formation of shear bands. The Clausing specimen was chosen because of its plane stress/plane strain deformation field, its familiarity with the current investigators and its amenability, because of geometry and size, to be tested in smaller capacity mechanical testing machines.

\section{Experimental Procedure}

The Clausing specimens were machined from a $50.8 \mathrm{~mm}$ by $50.8 \mathrm{~mm}$ cross-section hot rolled $70 / 30 \alpha$ brass bar. The specimens were machined in such a way that the direction of the tensile load was parallel to the rolling direction. The average grain size of the brass varied from 36 microns at the surface to 51 microns at the center of the hot rolled bars. The gage sections of the specimens were polished after machining using $240,320,400,600$ grit papers followed by 3 micron and 0.25 micron diamond paste to remove any surface notches or deep scratches to ensure that no surface effects affected the deformation of the specimen. 
Indentation markers were made using a microhardness tester as a reference for measuring displacements (Fig. 1). A clip gage was attached to the specimen by means of knife edges as a means to control the amount of strain during each increment of testing. The specimen was loaded uniaxially in an Instron mechanical testing machine to just above its yield point. The specimen was thereafter unloaded and the following measurements were made. The width of the specimen gage section was measured and so also the thicknesses at various points across the section. The displacement in the tensile direction was obtained by measuring the distance between the indentation markers using a measurescope. A photograph of the gage section was taken at 6.6X magnification using a stereomicroscope. A micrograph of the center of the gage section was also obtained using a metallurgical microscope at $100 \mathrm{X}$ and $400 \mathrm{X}$ magnifications. The specimen was again loaded in the mechanical testing machine and subjected to another small increment (1-2\%) in strain. All the above measurements were repeated after this strain increment. The above procedure was repeated for many increments until the specimen failed by fracture. The crosshead speed of the mechanical testing machine during tensile loading was $0.01 \mathrm{~cm}$ per minute. The low magnification photographs were taken with the aim of monitoring the surface deformation from a macroscopic viewpoint, whereas the micrographs were used to observe the micromechanical deformation processes and the possible evidence of shear band formation.Four different specimens were tested. The width and the thickness measurements were not made for specimen 3 , nor were the macrographs and the micrographs obtained for all cases. Temperature effects were not considered because the tests were carried out at room temperature. Table 1 is a compendium of relevant information about the specimens that were tested.

TABLE 1. Summary of Relevant Parameters for the Specimens Tested.

\begin{tabular}{ccccc}
\hline Specimen No. & Width W $(\mathrm{mm})$ & Thickness $\mathrm{t}(\mathrm{mm})$ & W/t & Avg. Grain Size $(\mu \mathrm{m})$ \\
\hline 1 & 25.45 & 2.16 & 11.78 & 51 \\
2 & 24.41 & 1.96 & 12.45 & 36 \\
3 & 25.37 & 2.01 & 12.62 & 36 \\
4 & 25.45 & 2.04 & 12.48 & 51 \\
\hline
\end{tabular}

\section{Results and Discussion}

The engineering stress-strain curves for the four specimens that were tested are depicted in Fig. 2. Each of the data points of the curve for a single specimen corresponds to one increment during testing. The engineering stress-strain curves for specimens 1 and 4 lie close to each other. The same is true for specimens 2 and 3 . This is to be expected because of the similar grain sizes for each pair of specimens (Table 1). There is a clear "dip" in these curves at an engineering straine $\approx 0.1$ as indicated in Fig. 2.

The double logarithmic true stress-strain values for all the specimens are plotted in Fig. 3. A linear fit with a single set of $\mathrm{K}$ and $\mathrm{n}$ values is not appropriate for this plot. For each specimen the plot is best described by three linear segments (three sets of $K$ and $n$ values) up to the value of strain corresponding to the load maximum. The third linear segment is not shown in the case of specimen 2 because of the lack of data points. No attempt is made at this time to describe the deformation behavior beyond the load maximum. The junctions of these linear segments will hereafter be mentioned as slope discontinuities (SD) and are denoted conveniently as SD I, SD II and SD III (see Fig. 3) occurring at approximate true strain values of 0.05, 0.10 and 0.18 respectively. The $\mathrm{K}$ and $\mathrm{n}$ values for the three linear segments of each specimen that was tested are shown in Table 2. The $n$ values of the first and second linear segments for specimen 1 and 4 are greater than those for 2 and 3 . This is attributed to the larger grain size of the former. A similar phenomenon is observed in a plot of relative width versus engineering strain (Fig. 4). Such deformation behavior has been observed before by Dahl and Rees (8) and Ollilainen (10) who have also used a triple-n fit to their double logarithmic stress-strain data up to uniform strain for steels.

Careful examination of the micrograph (Fig. 5a) shows the development of "longitudinal" bands at $\varepsilon \approx 0.05$ (SD I). These bands are elongated grains along the direction of the tensile loading axis. At this strain value, these bands are not distributed throughout the width of the specimen gage section but are found only at the center. Such a feature is believed to be a result of the lateral constraint beginning to take effect at SD I. During the initial stages of deformation of the Clausing specimen, a diffuse lateral necking is observed along the width of the gage section. Evidence of this is presented in Fig. 6 . This is also proof that a plane stress state is encountered in the initial stages of deformation. However, with increasing deformation a self-developing lateral constraint is seen because the lateral deformation in the gage section is now constrained by the rigid nature of the thicker grip sections of the Clausing specimen. After SD I there is a clear decrease in the amount of deformation 
TABLE 2 . $K$ and $n$ values for the various specimens.

\begin{tabular}{|c|c|c|c|c|c|c|}
\hline \multirow[t]{2}{*}{ Specimen No. } & \multicolumn{2}{|c|}{ Segment 1} & \multicolumn{2}{|c|}{ Segment 2} & \multicolumn{2}{|c|}{ Segment 3} \\
\hline & $\mathrm{K}_{1}(\mathrm{MPa})$ & $\mathrm{n}_{1}$ & $\mathrm{~K}_{2}(\mathrm{MPa})$ & $\mathrm{n}_{2}$ & $\mathrm{~K}_{3}(\mathrm{MPa})$ & $\mathrm{n}_{3}$ \\
\hline 1 & 358 & 0.11 & 395 & 0.14 & 493 & 0.24 \\
\hline 2 & 403 & 0.06 & 433 & 0.09 & & \\
\hline 3 & 399 & 0.05 & 446 & 0.09 & 626 & 0.23 \\
\hline 4 & 380 & 0.11 & 416 & 0.14 & 550 & 0.27 \\
\hline
\end{tabular}

along the width as can be seen in Fig. 4. This can also be observed in Fig. 6a where a half-moon is formed in the gage section at $\varepsilon \approx 0.05$. This feature is a result of the plane stress state which allows the unconstrained lateral necking and hence little deformation within the region of the half-moon.

After SD I, a combined plane stress/ plane strain state is encountered with the lateral constraint exerting more and more of a significant influence with increasing strain. At SD II $(\varepsilon=0.1)$, the lateral constraint is in full effect and the stress state at the specimen gage section is one of plane strain. As a result, the lateral geometrical constraint forces deformation predominantly along the tensile axis resulting in grain elongation in this direction which is seen in the formation of the above bands. The greater the degree of lateral constraint, the greater will be the extent of the plane strain field at the center of the specimen gage section and the more these longitudinal bands will spread across the width of the gage section. Evidence of this is seen in Fig. 5b where "longitudinal" band formation has spread more across the width of the specimen gage section.

SD III ( $\varepsilon=0.18$ ) corresponds to the "necking" instability at maximum load. This occurs when the lateral constraint allows minimal deformation along the width and hence forces major deformation in the thickness direction. As a result of the above, localized necking occurs in the thickness direction. At this point, the longitudinal bands at the center of the gage section seem to disintegrate mostly because of the formation of the neck resulting from the localized through-the-thickness deformation at the center (Fig. 5c). The final fracture begins at the neck and then propagates outward toward both sides of the gage section. A sequence of macrographs of the specimen surface were taken to show the evolution of topography with increasing deformation in the gage section (Fig. 6). A schematic diagram of similar behavior as observed by Valkonen(11) for an AISI 1090 spheroidized steel is also shown in Fig. 7. No attempt is made to characterize in detail the deformation behavior after maximum load. Another carefully planned set of experiments should be performed to document this post-necking behavior.

\section{Conclusions}

1. The deformation behavior up to the load maximum of $70 / 30 \alpha$ brass is defined well by a triple-n logarithmic stress-strain plot.

2. Three slope discontinuities were observed in the logarithmic stress-strain curve. SD I is related to the strain at which the lateral constraint becomes operative during deformation, SD II is explained to be the result of the full effect of the lateral constraint and SD III corresponds to the necking instability at maximum load.

3. The existence of a combined plane stress/plane strain field in the Clausing specimen is verified.

4. Post-necking behavior, which is not explained here must be carefully studied, as an extension of the current investigation.

\section{Acknowledgment}

The authors are grateful for the support of this research by the National Science Foundation under Grant Award MMS-9010437. Special thanks to Mr. W. Matias and Mr. O. Vargas for preparing the Clausing specimens.

\section{References}

1. J.R. Low, Jr. and T.R. Prater, Final Rep. on Plastic Flow of Aluminium Aircraft Sheet under Combined Loads-II, 
OSRD No. 4052, Pennsylvania State College, 1944.

2. J.H. Hollomon, Trans. AIME 162, 268-290 (1945).

3. J.R. Low, "Properties of Metals in Materials Engineering", ASM, Metals Park, Ohio, 1949.

4. G.W. Geil and N.L. Carwile, J. Res. NBS 45, 129-147 (1950).

5. D.V. Wilson and Y.A. Konnan, Acta Metall. 12, 617-628 (1964).

6. W.B. Morrison, Trans. ASM 59, 824-846 (1966).

7. M. Atkinson, Metall. Trans. 15A, 1185-1192 (1984).

8. W. Dahl and H. Rees, Arch. Eisenhüttenwes. 50, 401-406 (1979).

9. W. Dahl and H. Rees, Arch. Eisenhüttenwes, 51, 77-81 (1980).

10. V. Ollilainen, Lic. Tech. Thesis, Helsinki University of Technology, Espoo, (1980).

11. A.E. Valkonen, Ph.D. Dissertation. The Ohio State University. Columbus, OH, (1987).

12. D.P. Clausing, Int. J. Fract. Mech. 6, 71 (1970).

13. A.K. Chakrabarti and J.W. Spretnak, Metall. Trans. 6A, 733 (1975).
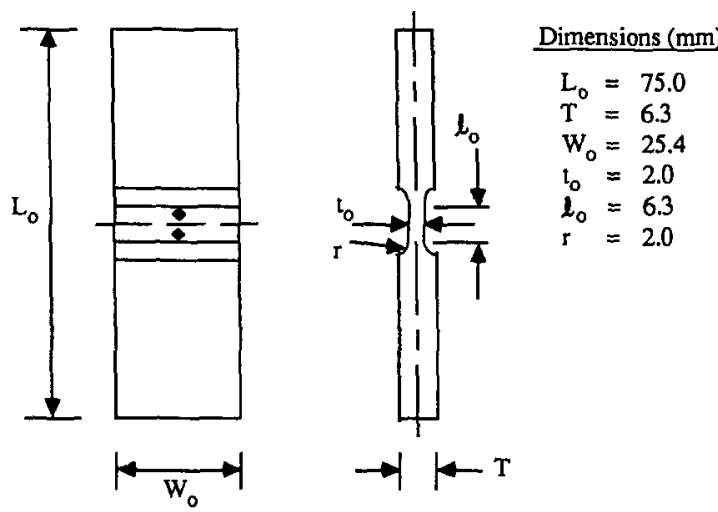

Fig. 1. Geometry and dimensions of the Clausing specimen. The tensile axis is vertical.

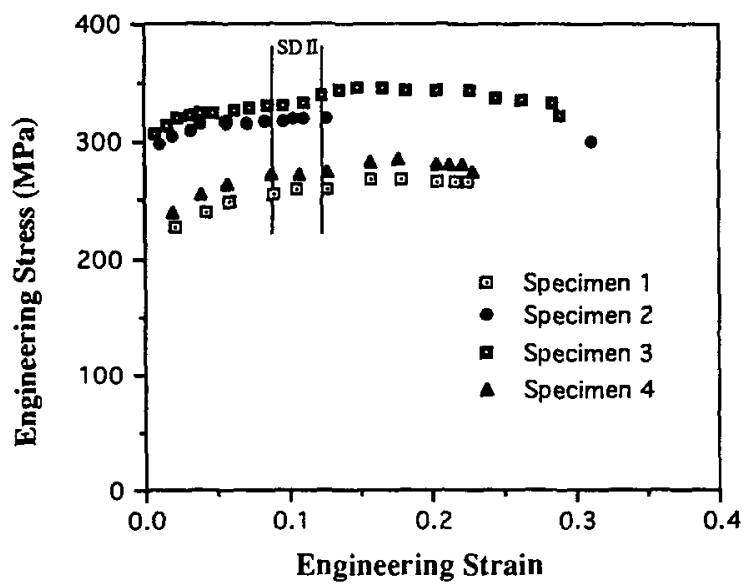

Fig. 2. Engineering stress-strain curves tor the various specimens. 


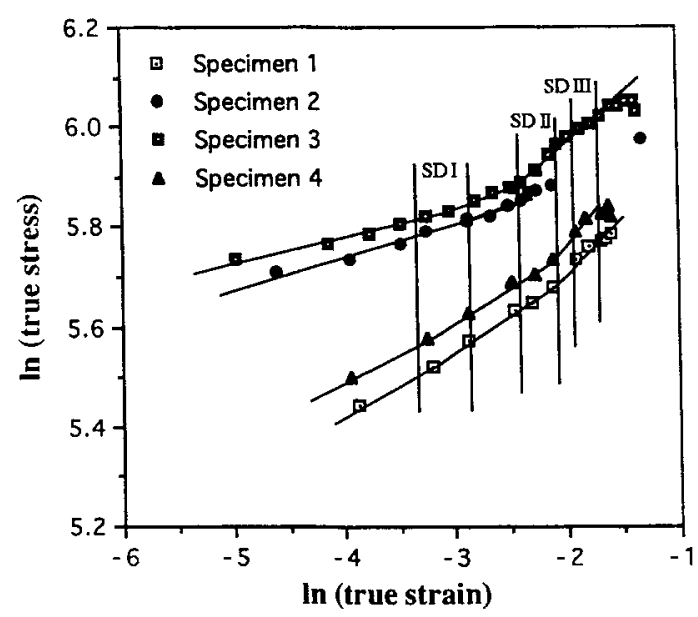

Fig. 3. Double logarithmic true stress-true strain plot for the various specimens.

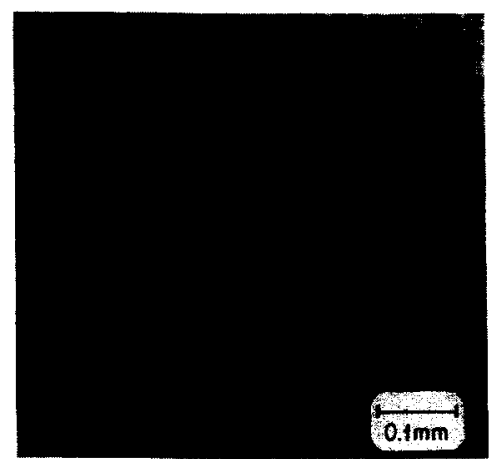

(a)

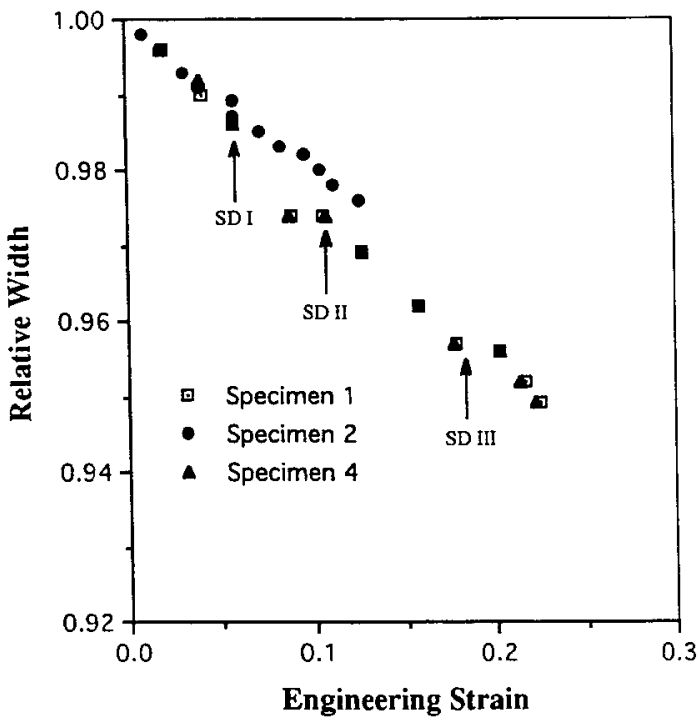

Fig. 4. Relative width versus engineering strain plots for the various specimens.

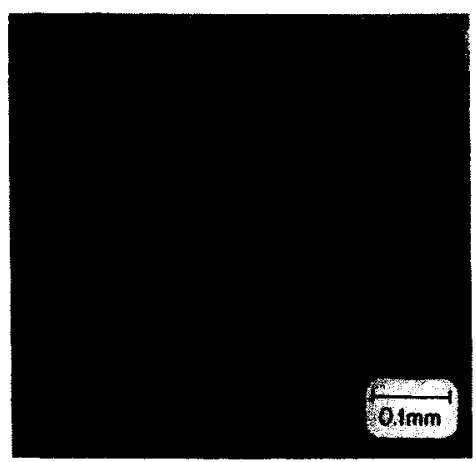

(b)

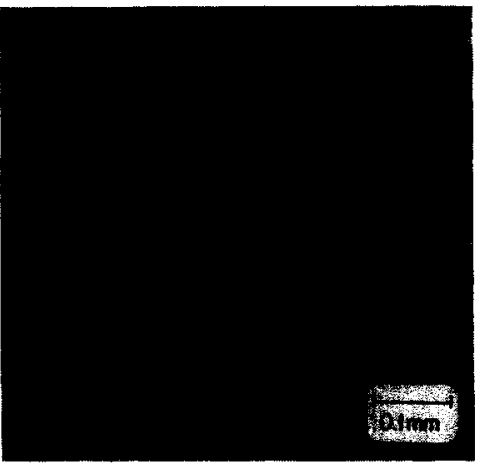

(c)

Fig. 5. Evolution of "longitudinal" bands (a) central bands at $\varepsilon=0.058$, (b) adjacent to the central band at $\varepsilon=0.1$ and (c) disintegration of bands due to localized necking. The tensile axis is horizontal. 


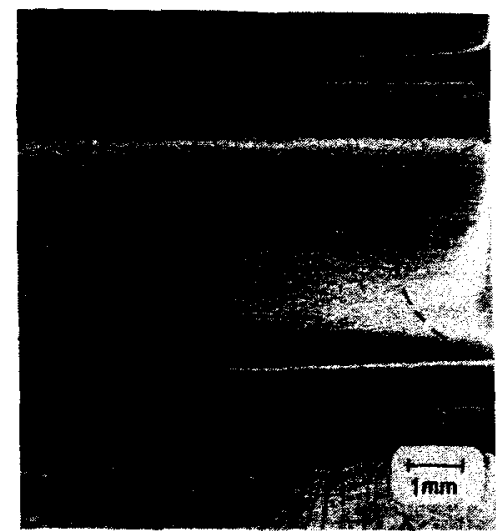

(a)

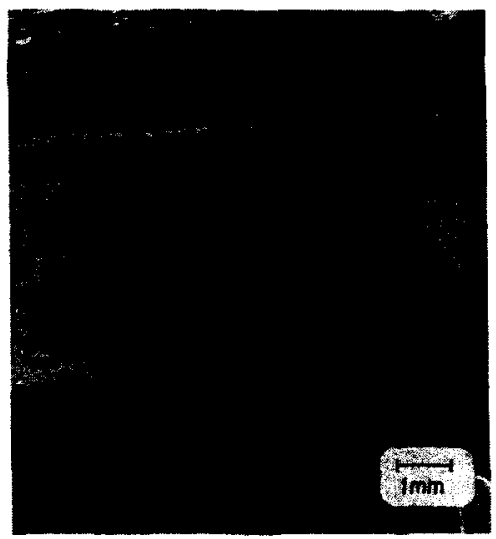

(c)

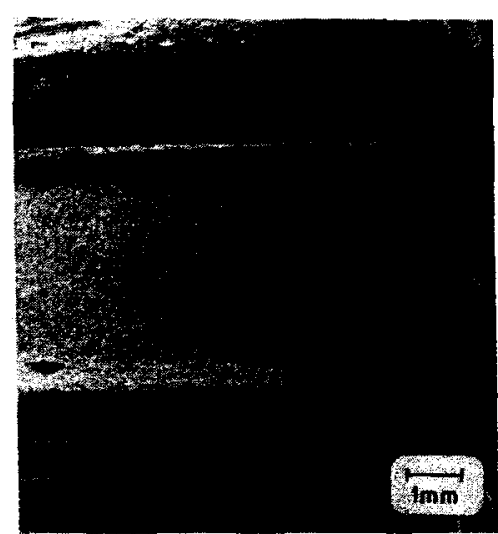

(b)

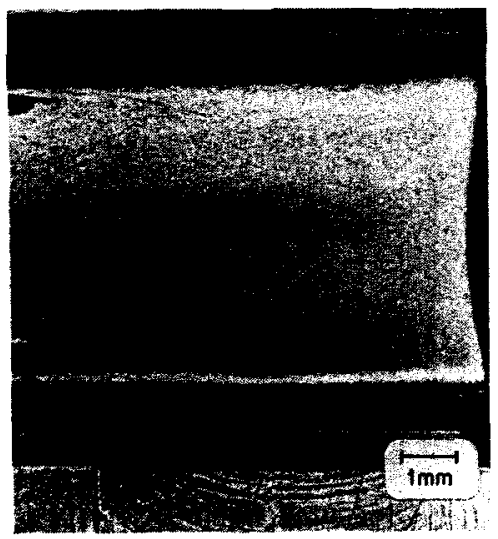

(d)

Fig. 6. Evolution of surface topography in the gage section at various strains. (a) $\varepsilon=0.058$, (b) $\varepsilon=0.113$, (c) $\varepsilon=0.162$ and (d) $\varepsilon=0.292$. The tensile axis is vertical.
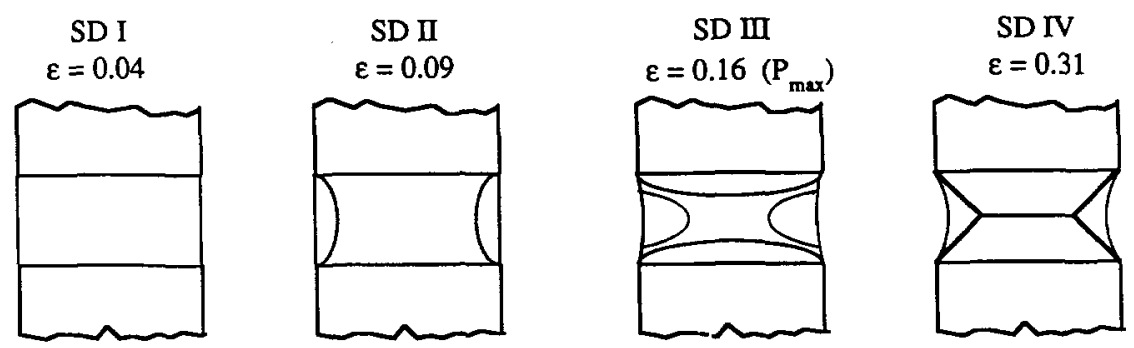

Fig. 7. Schematic diagram of surface topography evolution (11). The tensile axis is vertical. 